\title{
Avaliação da Efetividade das Atividades de Monitoria no Componente Curricular de Introdução à Programação
}

\author{
Leonardo Silva \\ ${ }^{1}$ Instituto Federal de Pernambuco \\ Garanhuns - PE - Brazil \\ leonardo.silva@garanhuns.ifpe.edu.br
}

\begin{abstract}
Teaching assistant (TA) activities are an educational practice frequently used in introductory programming courses to mitigate students' difficulties. Despite the apparent benefits, statistical data is needed to validate the pedagogical effectiveness of these actions. The present study is part of this context and investigates the association between students' academic performance and their presence in TA activities. The educational data of thirty-two students enrolled in a technical computing course was analyzed during the school year. A statistically significant association was found between programming learning and lower dropout with the students' frequency in TA activities. This research seeks to stimulate discussions on this topic, using statistical data as a reference.
\end{abstract}

Resumo. As atividades de monitoria são uma prática educacional utilizada com frequência em cursos de introdução à programação com o objetivo de mitigar dificuldades dos estudantes. Apesar dos aparentes benefícios, são necessários dados estatísticos que validem a efetividade pedagógica dessas ações. $O$ presente estudo se insere neste contexto e destina-se a investigar a associação entre o desempenho acadêmico do estudante com a sua presença em atividades de monitoria. Participaram do estudo trinta e dois estudantes matriculados em um curso técnico de computação integrado ao ensino médio. Foi identificada uma associação estatisticamente significante entre o desempenho em programação e menor evasão com a presença dos estudantes nas atividades de monitoria. $O$ estudo busca estimular discussões sobre esse tema, trazendo dados estatísticos que dão suporte à adoção das ações de monitoria.

\section{Introdução}

O aprendizado de programação é considerado complexo por muitos estudantes e professores [Qian and Lehman 2017, Gomes et al. 2012], resultando em elevados índices de evasão e retenção [Silva 2018]. Pesquisadores investigam as causas que impactam esse problema e como desenhar intervenções para mitigá-lo. Neste sentido, os atores participantes do processo educacional (estudantes, professores e instituições de ensino) possuem um papel ativo e devem realizar ações visando superar as dificuldades que cada um possui.

Ao escopo deste estudo interessa as ações de competência das instituições de ensino que devem oferecer apoio pedagógico aos seus alunos como forma de superar suas limitações. Um exemplo de ação são as atividades de monitoria, nas quais estudantes que já concluíram o componente curricular de programação dão suporte a outros alunos. Dentre os benefícios da monitoria, destaca-se o incentivo à interação entre estudantes, 
pois muitos argumentam que se sentem mais à vontade desta forma [Frison 2016]. Em cursos à distância a monitoria possui um papel ainda mais importante, pois possibilita uma interação social entre estudantes para além do espaço virtual [Silveira et al. 2018]. Este tipo de atividade auxilia na organização das rotinas de estudo, pois para muitos estudantes estes espaços representam sua única oportunidade de estudo [Nunes 2007]. As ações de monitoria também possibilitam ao professor receber feedback sobre a sua prática pedagógica e coletar dados sobre o desempenho da turma. Por fim, há uma contribuição sócio-econômica, tendo em vista que muitos estudantes monitores recebem bolsas para participar dessas ações. Essas ações de sócio-inclusão são especialmente eficazes para que estudantes em vulnerabilidade social não evadam [Matoso 2014].

Apesar dos aparentes benefícios e de ser amplamente utilizada pelas instituições de ensino, é preciso avaliar a efetividade das atividades de monitoria, tendo em vista que são empregados recursos financeiros e humanos para sua realização. Neste sentido, observa-se uma carência de dados estatísticos que validem essa prática em um contexto de ensino de programação [Silveira 2017]. Consequentemente, a avaliação sobre como os estudantes se beneficiam dessas ações é prejudicada, o que impacta a tomada de decisão de gestores educacionais que precisam justificar a realização dessas atividades. Observase que as discussões sobre essa temática são mais frequentes no contexto educacional de nível superior o que impacta a avaliação dessas políticas educacionais em outras modalidades de ensino, como os cursos técnicos da área de computação.

Diante do exposto, este estudo busca avaliar a efetividade de atividades de monitoria em um curso técnico de computação, em relação ao número de aprovados e evadidos no componente curricular de introdução à programação. Para isso foi realizada uma investigação ex-post-facto observacional com o uso de métodos quantitativos, que teve por objetivo responder às seguintes questões de pesquisa: a) A presença dos estudantes em atividades de monitoria está associada ao seu desempenho no componente curricular de introdução à programação? e b) A presença dos estudantes em atividades de monitoria está associada aos índices de evasão no componente curricular de introdução à programação?

Trinta e dois estudantes matriculados em um curso técnico de informática integrado ao ensino médio foram acompanhados ao longo de um ano letivo. Para responder as questões de pesquisa estabelecidas, testes estatísticos foram realizados considerando as médias finais dos estudantes no componente curricular de introdução à programação, suas frequências de participação nas atividades de monitoria e o estado do estudante ao término do ano letivo (abandono ou permanência no componente curricular). Para dar suporte à análise dos resultados também foram realizadas entrevistas com os alunos, monitores e professor do componente curricular.

\section{Revisão da Literatura}

Atividades de monitoria representam uma ação pedagógica de apoio aos discentes para que alcancem seus objetivos educacionais e êxito no processo educacional [Lins et al. 2009]. Em geral, essas ações são realizadas por discentes que já concluíram o componente curricular e são ofertadas no turno contrário ao ensino regular. De participação opcional, o estudante monitor utiliza esses espaços para tirar dúvidas de outros alunos e reforçar conteúdos vistos em sala de aula. 
Para muitos estudantes a monitoria representa um importante espaço de acolhimento, no qual podem tirar dúvidas que não conseguiram em sala de aula, seja por falta de tempo ou por timidez, como também praticar programação. A monitoria ajuda a criar uma rotina de estudos que para muitos alunos é a sua principal forma de estudo [Moreira et al. 2018]; [Holanda et al. 2018]; vivenciam momentos que ajudam na criação de laços afetivos entre estudantes e que funcionam como um suporte motivacional para que não desistam diante das dificuldades [Santana et al. 2017]. Isto é ainda mais relevante, pois o componente de introdução à programação apresenta elevados índices de reprovação [Silva 2018] e necessita que os estudantes estejam motivados para alcançar o sucesso acadêmico [Gomes et al. 2018].

A monitoria também oferece um importante suporte aos professores. Em razão do elevado quantitativo de alunos nas turmas, em muitos casos não conseguem oferecer feedback adequado a todos os estudantes e os monitores podem auxiliá-lo neste processo que é fundamental ao aprendizado [Hattie and Timperley 2007]. Para além disto, o estudante monitor consegue oferecer uma visão complementar sobre a turma que pode auxiliar o professor em sua tomada de decisão, como por exemplo, decidir sobre qual conteúdo precisa ser reforçado.

Em uma perspectiva teórica, atividades de monitoria são classificadas como uma prática educacional de cunho cooperativo fundada em uma perspectiva sócioconstrutivista [Frison 2016]. Sabe-se que a interação entre estudantes é fundamental ao processo de ensino e aprendizagem, pois permite que troquem experiências e recebam suporte para superar dificuldades que isoladamente não seriam capazes ou teriam grande dificuldade [Nunes 2007]. Percebe-se que, como discutido no conceito de Zona de Desenvolvimento Proximal de Lev Vygotsky, a interação social é uma base de aprendizagem para muitos estudante que necessitam de apoio para realizar certas atividades, e as atividades de monitoria apresentam essa característica [Cunha Júnior 2017].

A prática cooperativa e colaborativa é tida como um fato de sucesso para o processo de ensino e aprendizagem de programação [Silva et al. 2020]. A importância da monitoria para o aprendizado de programação é destacada por diferentes professores e pesquisadores [de Oliveira et al. 2017, Martins et al. 2010]. Estudos sobre essa temática destinam-se a compreender seus benefícios acadêmicos e entender como o processo decorre, considerando as especificidades do aprendizado de programação. Neste sentido, Silveira destacam que a maioria dos estudantes reprovados não participava de atividades de monitoria [Silveira 2017].

Por fim, os estudantes monitores são beneficiados com as atividades de monitoria. Gouveia et al. entrevistaram monitores de programação que relataram a importância dessas ações para o seu próprio crescimento educacional, valorização da experiência de compartilhar seus conhecimentos com outros estudantes e senso de responsabilidade e auto estima que são fundamentais para realização pessoal [Gouveia et al. 2018]. Resultados similares também são apresentados por Figuerêdo et al., corroborando que as ações de monitoria impactam positivamente os múltiplos atores que fazem parte deste processo [Figuerêdo et al. 2021].

Com exceção de [Silveira 2017], os trabalhos realizam análises qualitativas sobre as atividades de monitoria em introdução à programação. Apesar dos seus benefícios e 
de um "senso comum"sobre as vantagens em realizar essas atividades, há a necessidade de prover mais evidências estatísticas sobre sua efetividade. Para além disto, os estudos supradescritos foram realizados em um contexto educacional de nível superior e o presente trabalho busca ampliar o conhecimento considerando estudantes do ensino técnico de computação.

\section{Metodologia}

Este estudo caracteriza-se como exploratório ex-post-facto e observacional que tem por objetivo investigar as diferenças nos dados acadêmicos de estudantes que frequentam e não frequentam atividades de monitoria de programação.

\subsection{Descrição da amostra}

A amostra deste estudo é composta por trinta e dois estudantes matriculados em um curso técnico de computação integrado ao ensino médio do Instituto Federal de Pernambuco - Campus Garanhuns. Neste curso é ofertada qualificação profissional para que os egressos atuem no segmento de desenvolvimento de software. A formação ocorre concomitantemente ao período em que cursam o ensino médio e de forma integrada ao seu currículo. Esta modalidade de ensino profissionalizante possui duração de 4 anos, na qual os estudantes possuem componentes curriculares tradicionais do ensino médio, como matemática, português, física, dentre outros, mas também componentes de computação, como introdução à programação, análise orientada a objetos, programação para a Internet, testes de software, entre outros, ao longo de todo o curso.

Os dados coletados representam o período de um ano letivo. À época em que cursaram o componente curricular de introdução à programação, os estudantes apresentavam faixa-etária entre 14 e 16 anos e há uma distribuição equivalente entre os gêneros. Aproximadamente $60 \%$ dos estudantes recebem bolsas de assistência social em razão de sua vulnerabilidade sócio-econômica. $98 \%$ não possuíam experiência prévia em programação e aproximadamente $30 \%$ não possuíam computador em casa, sendo o campus a única oportunidade para prática da programação. Esse último dado foi fundamental para o delineamento desse estudo, pois muitos estudantes expressaram a necessidade do campus oferecer computadores e apoio pedagógico no contra turno para realização dos seus estudos em programação. Nesse sentido, foram ofertadas atividades de monitoria.

\subsubsection{Contexto Pedagógico}

O componente curricular de introdução à programação está presente no primeiro ano de curso e dispõe de uma carga horária semanal de 2 horas, totalizando 80 horas ao término do ano letivo. O conteúdo curricular contempla: pensamento algorítmico, variáveis, tipos de dados, estruturas de controle de fluxo e repetição, vetores e funções, ministrados com a linguagem de programação Lua e Java. As aulas ocorreram em um laboratório de informática com a disponibilidade de um computador por estudante. O professor deste componente curricular, em geral, utilizou uma metodologia de ensino expositiva, intercalada com atividades práticas de laboratório. Quatro avaliações foram realizadas ao longo do ano, sendo uma por bimestre e com direito a recuperação. As atividades avaliativas foram realizadas com papel e caneta e os alunos deveriam construir algoritmos para 
problemas que eram passados. Historicamente na instituição o índice de reprovação no componente curricular é de aproximadamente 50\% [Silva 2018].

A partir do terceiro mês letivo os estudantes passaram a ter suporte de dois monitores, sendo um bolsista e outro voluntário. Os monitores deram suporte ao professor durante as atividades em laboratório, em especial apoiando os estudantes na criação de algoritmos. Foi estabelecido um encontro semanal de 2 horas, denominado plantão de dúvidas, onde o monitor bolsista resolvia dúvidas dos estudantes, listas de exercícios passadas pelo professor e reforçava eventuais conteúdos solicitados pelo professor ou alunos.

\subsection{Coleta dos Dados}

Três dados foram fundamentais para a realização do cálculo estatístico deste estudo: i) o quantitativo de presenças dos estudantes no encontro semanal com o monitor bolsista; ii) o desempenho em programação que foi mensurado pelo resultado final do estudante no componente curricular, sendo aprovado (média igual ou superior a seis) ou reprovado; e iii) o percentual de estudantes evadidos do componente curricular que foi calculado ao término do ano letivo. $\mathrm{O}$ estudante que não realizou as atividades avaliativas do terceiro e quarto bimestre foi considerado evadido. Por fim, no decorrer do ano letivo alguns estudantes foram entrevistados para avaliar suas percepções sobre as atividades de monitoria. Ao término do ano letivo os estudantes monitores e o professor relataram suas experiências.

\subsection{Planejamento estatístico}

Considerando as questões de pesquisas que foram definidas e as informações coletadas, optou-se pelo uso do teste de Qui-Quadrado de Pearson, pois ele possibilita calcular associação de dependência entre as variáveis do estudo [McHugh 2013]. Portanto, verificou-se a associação entre o resultado final no componente curricular (aprovado ou reprovado) e a situação do estudante (evadido ou não evadido) com a sua participação nas atividades de monitoria. Essa participação foi agrupada em dois grupos: a) 50\% ou mais de presença; e b) menos que $50 \%$ de presença. Optou-se por este número por entendermos que a falta do estudante em mais de metade das atividades poderia comprometer o seu desempenho escolar. A base para o cálculo do Qui-Quadrado consiste na construção de uma tabela de contingência, em que as variáveis analisadas são dispostas em linhas e colunas, construindo assim associações. Após isto contabiliza-se a frequência das ocorrências de cada associação, como apresentado na Tabela 1. Como o resultado do teste apenas indica a existência de associação entre as variáveis, também foram calculados os tamanhos de efeito (effect-size) para verificar a intensidade da influência de uma variável sobre a outra. Para o contexto deste estudo, esse dado indica o quanto a presença nas atividades de monitoria contribuíram para o desempenho em programação e nos índices de evasão. Utilizou-se a fórmula de Cramer's V para calcular o tamanho de efeito entre as variáveis, que resulta em um valor dentro de uma escala de 0 a 1 [Coe 2002].

\section{Resultados}

Para representar a associação entre o desempenho em introdução à programação e evasão com a frequência dos estudantes nas atividades de monitoria foi construída uma tabela de contingência 1. Nela, apresenta-se o total de estudantes aprovados e reprovados, evadidos e não evadidos, agrupados pela frequência nas atividades de monitoria. 
Tabela 1. Associação entre o desempenho acadêmico e número de evadidos com a frequência na monitoria.

\begin{tabular}{|l|l|l|l|l|}
\hline & Aprovado & Reprovado & Evadido & $\begin{array}{l}\text { Não eva- } \\
\text { dido }\end{array}$ \\
\hline $\begin{array}{l}\text { Frequência igual ou maior } \\
\text { que 50\% }\end{array}$ & 10 & 2 & 0 & 12 \\
\hline Frequência menor que 50\% & 6 & 14 & 10 & 10 \\
\hline
\end{tabular}

O cálculo de Qui-Quadrado de Pearson entre o desempenho acadêmico e a presença na monitoria resultou em: $\chi 2=6,5333$, graus de liberdade $=1 \mathrm{e} p$-value $=$ 0,01 . Enquanto que o cálculo de associação entre os índices de evasão e a presença na monitoria resultou em: $\chi 2=6,5552$, graus de liberdade $=1 \mathrm{e} \mathrm{p}$-value $=0,01$. Os valores de p identificados para um nível de significância estatística de 95\%, indicam que os resultados são estatisticamente significantes. Portanto, calculou-se o tamanho de efeito para a associação de dependência entre o desempenho em programação e a presença nas atividades de monitoria que resultou em 0,451 ; e com o índice evasão o resultado foi de 0,452 .

Nas entrevistas, os estudantes avaliaram positivamente as atividades de monitoria, ressaltando sua contribuição para a compreensão de conceitos mais complexos da programação. Também relataram que o acesso aos laboratórios foi fundamental ao aprendizado, tendo em vista que alguns não possuíam computador ou acesso à Internet em suas residências. Os estudantes monitores avaliaram positivamente a experiência para a sua formação. Alguns obstáculos foram relatados, em especial sobre aspectos pedagógicos. Para eles, conseguir intervir para mitigar as dificuldades dos alunos foi o maior desafio, pois nem sempre o que faziam provocava melhorias. O professor do componente curricular expôs a importância dos estudantes monitores, em especial nas atividades em laboratório. Em razão do número de estudantes no laboratório, relatou que não conseguia oferecer um acompanhamento e feedback adequado, e que com a presença dos monitores conseguiu distribuir essa atividade que em sua avaliação foi fundamental ao processo de ensino e aprendizagem. Além disto, ao término de cada aula, os monitores relatavam suas percepções, indicando estudantes que possuíam maiores dificuldades e apoiavam com sugestões sobre melhorias. Essa perspectiva foi considerada positiva pelo docente.

\section{Análise dos Resultados}

Pela Tabela 1 observa-se que os estudantes que frequentaram mais de cinquenta por cento das atividades de monitorias obtiveram melhores resultados no componente curricular e apresentaram menor evasão. Dos estudantes com maior frequência nas atividades de monitoria $(n=12), 83,3 \%$ foram aprovados e nenhum evadiu. Dos estudantes que frequentaram menos de cinquenta por cento das atividades de monitoria $(n=20)$ houve $30 \%$ de aprovações e 50\% de evasão. Os testes de Qui-Quadrado de Pearson indicaram a existência de uma associação de dependência entre desempenho e evasão com a presença nas atividades de monitoria ( $\mathrm{p}$-value $=0,01$ ). Os cálculos de tamanho de efeito indicam a existência de uma associação de intensidade média $(>0,4)$ [Coe 2002], que para pesquisas educacionais é considerado como uma influência relevante [Fan 2001]. Os dados corroboram os achados de Silveira sobre a importância da participação dos estudantes de 
programação nas atividades de monitoria [Silveira 2017].

Para interpretar os resultados descritos convém ressaltar o contexto educacional deste estudo. Aproximadamente $60 \%$ dos estudantes do campus recebem bolsa de assistência estudantil, pois possuem uma condição sócio-econômica vulnerável. Muitos não possuem computador e/ou Internet para estudar em suas residências. Como relatado por esses alunos, o espaço de monitoria permitia praticar as atividades de programação, pois em suas casas não possuíam a estrutura necessária. Além disso, alguns alunos mencionaram que possuíam dificuldades em organizar suas rotinas de estudo e que a monitoria os ajudava à dedicarem um tempo para os estudos.

Pelos relatos dos estudantes entrevistados, avalia-se que um dos benefícios da monitoria é proporcionar um momento de interação social, para além do espaço de sala de aula. O elemento motivacional foi observado nas respostas, acredita-se que as interações extrapolam o escopo educacional e servem de suporte motivacional, um dos aspectos que pode contribuir com a redução dos índices de evasão [Khalkhali et al. 2013]. No entanto, pondera-se que esse último elemento carece de investigações futuras.

Foi observado que a maior parte dos estudantes matriculados no componente curricular $(62,5 \%)$ não frequentou ou teve menos de $50 \%$ de presença nas atividades de monitoria. Este problema também foi observado em outros estudos [Moreira et al. 2018, dos Santos Silva et al. 2018]. Os motivos apresentados são diversos, incompatibilidade de horários, transporte, entre outros. Em casos mais sérios, os estudantes não visualizam a importância desses espaços para seu aprendizado [Oliveira et al. 2017]. No contexto deste estudo essas causas não foram investigadas e devem ser exploradas em trabalhos futuros.

Os estudantes monitores relataram sua realização pessoal em contribuir com outros estudantes, além das relações interpessoais que foram desenvolvidas. Observou-se certa dificuldade dos monitores em identificar as dificuldades dos estudantes, apoiá-los durante o aprendizado e realizar o plantão de dúvidas. Para mitigar essas dificuldades sugere-se a inclusão de programas de formação pedagógica para os monitores. Além disso, também é fundamental que a instituição de ensino e professores façam acompanhamentos rotineiros das atividades desempenhadas pelos monitores para garantir que estão sendo realizadas com qualidade e que toda a estrutura necessária para sua realização está sendo provida [de Oliveira et al. 2017].

Pelo relato do professor, observa-se que as atividades de monitoria deram suporte ao alcance dos objetivos educacionais estabelecidos para o componente curricular, como também na oferta de feedback educacional adequado. O professor destacou que os monitores desenvolveram habilidades que podem contribuir para sua formação educacional e atuação profissional, como o desenvolvimento das relações interpessoais.

Pondera-se os resultados em relação ao tamanho da amostra analisada e ao fato de que estudos associativos não podem explicar uma relação causalística [McHugh 2013]. Disto isto, avalia-se o resultado encontrado pode servir de suporte para que novos estudos para ampliem o corpo de evidências sobre essa temática.

Por fim, tudo o que foi apresentado precisa ser analisado considerando a complexidade do processo de ensino e aprendizagem de programação. Há inúmeros fatores que contribuem positivamente e negativamente, e não pode-se afirmar que apenas a presença 
nas atividades de monitoria resultará em sucesso acadêmico para os estudantes. Para além disto, sabe-se que a metodologia adotada pelos professores, sua formação continuada, a oferta de laboratórios de informática com quantitativo suficiente de computadores, rotina de estudo dos alunos, suas habilidades cognitivas e metacognitivas, e o suporte motivacional, impactam o aprendizado de programação. Por exemplo, Silva e Cavalcanti [Silva and Cavalcanti 2018] relatam que tecnologias educacionais podem ser utilizadas para reduzir os índices de evasão em programação. Assim, um conjunto de ações são necessárias e devem ser consideradas para aumentarem as chances de êxito dos estudantes, para além das atividades de monitoria.

\section{Limitações do estudo}

Estudos observacionais são limitados quanto à capacidade de associar conclusões aos fatores observados [Gersten et al. 2000]. Neste sentido, ponderamos que os resultados apresentados não implicam em uma relação de causalidade. Destaca-se também o contexto educacional deste estudo que envolveu estudantes adolescentes de um curso técnico. Assim, também deve-se investigar o impacto das ações de monitoria com estudantes de nível superior. Reforça-se que aprendizado de programação é influenciado por múltiplos fatores, além do tamanho das turmas [Medeiros et al. 2018], que também podem impactar no resultado. Por fim, o tamanho da amostra é outro fator limitante quanto à generalização dos resultados. Estudos futuros devem replicar a metodologia adotada nessa investigação para ampliar o corpo de conhecimento sobre o tema.

\section{Conclusão}

Muitos estudantes de programação apresentam dificuldades durante o aprendizado. As atividades de monitoria podem servir de apoio pedagógico para que estes estudantes alcancem o êxito educacional, no entanto, a literatura atual carece de evidências estatísticas sobre a eficácia dessa atividade pedagógica. O presente estudo teve por objetivo avaliar como a presença de estudantes nas atividades de monitoria está associada ao número de aprovações e evasões no componente curricular de introdução à programação. Para a amostra analisada, os resultados demonstram que estudantes com maior frequência nas atividades de monitoria alcançaram melhor desempenho acadêmico. Trabalhos futuros podem replicar a metodologia utilizada neste estudo para construir um corpo de conhecimento sobre essa temática, em especial com outras modalidades de ensino, como o nível superior.

\section{Referências}

Coe, R. (2002). It's the effect size, stupid: What effect size is and why it is important.

Cunha Júnior, F. R. d. (2017). Atividades de monitoria: uma possibilidade para o desenvolvimento da sala de aula. Educação e Pesquisa, 43(3):681-694.

de Oliveira, B. P., Balan, G. R., Leitao, P., and Coutinho, E. F. (2017). Identificação e discussão de problemas nas disciplinas iniciais de programação do curso de graduação sistemas e mídias digitais. Revista Sistemas e Mídias Digitais (RSMD), 2(1).

dos Santos Silva, W., Lima, M. S., dos Santos Raposo, J. C., and da Silva Júnior, L. C. F. (2018). Levantamento sobre as dificuldades dos discentes nas disciplinas de programação no curso técnico de informática. Diversitas Journal, 3(3):761-770. 
Fan, X. (2001). Statistical significance and effect size in education research: Two sides of a coin. The Journal of Educational Research, 94(5):275-282.

Figuerêdo, J. S. L., Machado, J. G., Lima, S. V., da Silva Cerqueira, C. S., and Pereira, C. P. (2021). A experiência da monitoria de algoritmos e programação em cursos de engenharia na perspectiva dos monitores. In Anais do Simpósio Brasileiro de Educação em Computação, pages 183-192. SBC.

Frison, L. M. B. (2016). Monitoria: uma modalidade de ensino que potencializa a aprendizagem colaborativa e autorregulada. Pro-Posições, 27(1):133-153.

Gersten, R., Baker, S., and Lloyd, J. W. (2000). Designing high-quality research in special education: Group experimental design. The Journal of Special Education, 34(1):2-18.

Gomes, A., Ke, W., Lam, C.-T., Marcelino, M. J., and Mendes, A. (2018). Student motivation towards learning to program. In 2018 IEEE Frontiers in Education Conference (FIE), pages 1-8. IEEE.

Gomes, A. J., Santos, A. N., and Mendes, A. J. (2012). A study on students' behaviours and attitudes towards learning to program. In Proceedings of the 17th ACM annual conference on Innovation and technology in computer science education, pages 132137.

Gouveia, S. S. D. S., Vasconcelos, N. V. C. D., and Palhares, R. d. A. (2018). Análise da metodologia de ensino da disciplina de programação de computadores sob o ponto de vista dos monitores. XVIII Colóquio Internacional de Gestão Universitária.

Hattie, J. and Timperley, H. (2007). The power of feedback. Review of educational research, 77(1):81-112.

Holanda, W., Coutinho, J., and Fontes, L. (2018). Uma intervenção metodológica para auxiliar a aprendizagem de programação introdutória: um estudo experimental. In Anais dos Workshops do Congresso Brasileiro de Informática na Educação, volume 7, page 699 .

Khalkhali, V., Sharifi, R., and Nikyar, A. (2013). Students' intentions to persist in, versus dropout of high school: What self-determined motivation tells us about it? International Online Journal of Educational Sciences, 5(2).

Lins, L. F., Ferreira, L. M. C., Ferraz, L. V., and CARVALHO, S. d. (2009). A importância da monitoria na formação acadêmica do monitor. Jornada de ensino, pesquisa e extensão, IX.

Martins, S. W., Mendes, A. J., and de Figueiredo, A. D. (2010). Comunidades de investigação em programação: Uma estratégia de apoio ao aprendizado inicial de programação. Rev. Iberoam. de Tecnol. del Aprendiz., 5(1):39-46.

Matoso, L. M. L. (2014). A importância da monitoria na formação acadêmica do monitor: um relato de experiência. CATUSSABA-ISSN 2237-3608, 3(2):77-83.

McHugh, M. L. (2013). The chi-square test of independence. Biochemia medica: Biochemia medica, 23(2):143-149.

Medeiros, R. P., Ramalho, G. L., and Falcão, T. P. (2018). A systematic literature review on teaching and learning introductory programming in higher education. IEEE Transactions on Education, 62(2):77-90. 
Moreira, G. L., Holanda, W., Coutinho, J. C. d. S., and Chagas, F. S. (2018). Desafios na aprendizagem de programação introdutória em cursos de ti da ufersa, campus pau dos ferros: um estudo exploratório. Anais do Encontro de Computação do Oeste Potiguar ECOP/UFERSA (ISSN 2526-7574), (2).

Nunes, J. B. C. (2007). Monitoria acadêmica: espaço de formação. A monitoria como espaço de iniciação à docência: possibilidades e trajetórias. Natal: EDUFRN, pages $45-58$.

Oliveira, M. A. F., de Lima, J. V., do Canto Filho, A. B., Nunes, F. B., Lourega, L. V., and Melo, J. N. B. (2017). Aplicação do método peer instruction no ensino de algoritmos e programação de computadores. RENOTE-Revista Novas Tecnologias na Educação, $15(1)$.

Qian, Y. and Lehman, J. (2017). Students' misconceptions and other difficulties in introductory programming: A literature review. ACM Transactions on Computing Education (TOCE), 18(1):1-24.

Santana, B. L., Figuerêdo, J. S. L., and Bittencourt, R. A. (2017). Motivaçao de estudantes non-majors em uma disciplina de programaçao. In Anais do XXV Workshop sobre Educação em Computação. SBC.

Silva, L., Mendes, A. J., and Gomes, A. (2020). Computer-supported collaborative learning in programming education: A systematic literature review. In 2020 IEEE Global Engineering Education Conference (EDUCON), pages 1086-1095. IEEE.

Silva, L. S. (2018). Análise do aprendizado em programação de estudantes do ensino técnico integrado do instituto federal de pernambuco. In Anais do V Encontro Nacional de Computação dos Institutos Federais. SBC.

Silva, L. S. and Cavalcanti, E. R. (2018). Avaliaçao experimental do robomind no ensino de programaçao com estudantes do curso técnico em informática integrado ao ensino médio. In Brazilian Symposium on Computers in Education (Simpósio Brasileiro de Informática na Educação-SBIE), volume 29, page 288.

Silveira, M. A. (2017). Análise das variáveis que influenciam no desempenho dos alunos nas disciplinas de programação. Master's thesis, Universidade Federal de Ouro Preto - UFOP.

Silveira, S. R., Pereira, A. S., Bertolini, C., Parreira, F., and Cunha, G. (2018). Potencialidades e desafios do curso de licenciatura em computação a distância da ufsm/uab. In Anais dos Workshops do Congresso Brasileiro de Informática na Educação, volume 7, page 1061 . 